\title{
GMR
}

\section{Therapeutic effect of Pleurotus eryngii cellulose on experimental fatty liver in rats}

\author{
J.F. Huang, T. Zhan, X.L. Yu, Q.A. He, W.J. Huang, L.Z. Lin, \\ Y.T. Du and Y.T. Pan \\ Engineering Technological Center of Mushroom Industry, \\ Minnan Normal University, Zhangzhou, Fujian, China \\ Corresponding author: Y.T. Pan \\ E-mail: panyutian_pyt@163.com \\ Genet. Mol. Res. 15 (1): gmr.15017805 \\ Received October 8, 2015 \\ Accepted December 4, 2015 \\ Published February 26, 2016 \\ DOI http://dx.doi.org/10.4238/gmr.15017805
}

\begin{abstract}
The aim of this study was to explore the therapeutic effect of Pleurotus eryngii cellulose on experimental fatty liver in rats. Rats were fed high-fat fodder to establish a rat fatty liver model, and were then fed different concentrations of Pleurotus eryngii cellulose for six weeks. Lipitor was used as a positive control. Measured levels of alanine aminotransferase (ALT), aspartate aminotransferase (AST), total cholesterol (TC), and total triglyceride (TG); the activity of malondialdehyde (MDA), superoxide dismutase (SOD), hepatic lipase (HL), and lipoprotein lipase; and liver histopathological changes. Successfully established rat fatty liver model after feeding high-fat fodder for one week. A diet of $P$. eryngii cellulose for six weeks significantly reduced ALT, AST, TC, and TG levels in rat serum $(P<0.01)$; TC and AST levels in P. eryngii cellulose high-dose group and Lipitor group were not significantly different from those of the control $(P>0.05)$. SOD activity increased significantly, while MDA and $H L$ activity decreased $(P<0.05)$; fatty degeneration and fat accumulation both decreased in hepatic tissue. Hepatic protection of $P$. eryngii cellulose showed dose-related effect. $P$. eryngii cellulose can affect lipid metabolism, having therapeutic effects on fatty liver in rats.
\end{abstract}

Key words: Pleurotus eryngii; Cellulose; Experimental fatty liver; Treatment 


\section{INTRODUCTION}

Pleurotus eryngii belongs to Pleurotus. $P$. eryngii is an edible mushroom with a fleshy, crisp texture, and the fragrance of abalone and almond. Per $100 \mathrm{~g}$, $P$. eryngii contains protein $16.2 \mathrm{~g}$, fat $3.8 \mathrm{~g}$, total carbohydrate $64.7 \mathrm{~g}$, calories $283 \mathrm{~J}$, cellulose $6.6 \mathrm{~g}$, ash $0.3 \mathrm{~g}$, potassium $262.7 \mathrm{mg}$, phosphorus $260 \mathrm{mg}$, magnesium $123.3 \mathrm{mg}$, calcium $26 \mathrm{mg}$, iron $3.2 \mathrm{mg}$, sodium $25.3 \mathrm{mg}$, vitamins B1 and B2, and other ingredients (Lee et al., 2009), making it a high-protein, low-fat, high-fiber product that is beneficial to human health. It can inhibit hydrolyzation of methylumbelliferone oleate and emulsification of glycerol trioleate, and reduce absorption of fat, thereby decreasing triglyceride (TG) and total cholesterol (TC) levels (Mizutani et al., 2010), and reduce atherosclerotic lesions (Mori et al., 2008). It can adjust T-helper activity, promote cytokine production, reduce infiltration of inflammatory cells and mastocytes, and reduce allergic dermatitis (Ike et al., 2012, Choi et al., 2013). It can inhibit the growth of Helicobacter pylori, Escherichia coli, Staphylococcus aureus, and Listeria monocytogenes (Shang et al., 2013; Li and Shah, 2014), and fight viruses such as influenza virus H1N1 and herpes simplex virus (Krupodorova et al., 2014).

The current definition of dietary fiber comes from American Chemical Society in 2001: the sum of edible vegetable ingredients, carbohydrates and similar substances that are resistant to digestion and absorption in the human small intestine, but can be partially or fully fermented in the human large intestine, including polysaccharides, oligosaccharides, lignin, and associated plant substances ( $\mathrm{Li}$ and Yang, 2007). Fat can adsorb to dietary fiber and be excreted with it, thereby reducing the absorption of fat and contributing to the prevention and cure of obesity and related disorders (Wang et al., 2009). Can P. eryngii cellulose, used as a dietary fiber, cure fatty liver? This study could provide the theoretical and scientific basis for practical application, developing into natural medicine and functional foods and expanding the traditional chain industry of $P$. eryngii.

\section{MATERIAL AND METHODS}

\section{Materials}

Male SD rats (cleaning grade) weighing $100 \pm 20 \mathrm{~g}$ were purchased from Shanghai Slack Laboratory Animal Co., Ltd., animal license number, SCXK (Shanghai) 2012-0002, and were housed separately for seven days at an ambient temperature of $22^{\circ} \pm 2^{\circ} \mathrm{C}$, with 12-h light/dark cycles, allowed to freely feed and drink water.

Fodder: high-fat fodder $(78.8 \%$ basal fodder, $1 \%$ cholesterol, $10 \%$ egg yolk powder, $10 \%$ lard, $0.2 \%$ cholate) and basal fodder were purchased from Shanghai Slack Laboratory Animal Co., Ltd.

Reagents: P. eryngii cellulose (PC) was prepared by Fungus Industrial Engineering Technology Center, Minnan Normal University. Lipitor was purchased from Pfizer; alanine aminotransferase (ALT), aspartate aminotransferase (AST), TC, and TG kits were purchased from Shenzhen Mindray Bio-Medical Electronics Co., Ltd.; superoxide dismutase (SOD), malondialdehyde (MDA), hepatic lipase (HL), and lipoprotein lipase (LPL) kits were provided by Nanjing Jiancheng Biology Engineering Institute.

Instruments: UV-visible spectrophotometer (UV-1100 MAPADA), Potter-Elvehjem Tissue 
Grinders (PRO200 PRO Scientific), ultra-low temperature freezer (Forma $-86^{\circ} \mathrm{C}$ Thermo), highspeed refrigerated centrifuge (5810R Eppendorf), biological phase-contrast microscope (BX51 OLYMPUS), electronic analytical balance (AUY120 SHIMADZU), automatic biochemical analyzer (BS-220 Mindry), histotome (5062 SLEE), tissue embedding machine (EG1150 LEICA), tissue processor (TP 1020 LEICA), Minishaker (MS1 Minishaker).

\section{Methods}

\section{Grouping and modeling}

Forty-eight male SD rats, fed continuously for one week to adapt to laboratory environment, were weighed and randomly divided into six groups of eight: control group (CG), model group (MG), Lipitor group (LG), P. eryngii cellulose high-dose group (PCHG), P. eryngii cellulose middledose group (PCMG), P. eryngii cellulose low-dose group (PCLG). The control group was given basic fodder, while the five model groups were fed with high fat fodder to establish the fatty liver model. Daily living conditions were observed; all rats were regularly weighed and eating habits were recorded. Intragastric administration of PC occurred daily at 7:00 p.m., after $2 \mathrm{~h}$ of fasting. After one week of feeding on high-fat fodder, random blood samples were drawn from the caudal veins of CG and MG rats and centrifuged ( $3000 \mathrm{rpm}, 4^{\circ} \mathrm{C}$ for $5 \mathrm{~min}$ ). The supernatant was separated, and AST, ALT, TG, and TC content in the serum were measured by automatic biochemical analyzer to establish fatty liver model.

\section{Administration method and dosage}

After modeling, the control group continued with basic fodder, while the five model groups continued to feed with high-fat fodder and daily intragastric administration of PC or Lipitor according to body weight ratio (10 mg/kg), LG (5 mg/kg per day), PCHG (1 g/kg per day), PCMG ( $0.5 \mathrm{~g} / \mathrm{kg}$ per day), PCLG $(0.25 \mathrm{~g} / \mathrm{kg}$ per day). The control group and model group were given the same dose of normal saline once daily for six weeks.

\section{Observation of indicators and measurement methods}

After the last administration, the rats fasted for $12 \mathrm{~h}$. They were weighed and blood was drawn from the caudal vein. TG, TC, AST, and ALT content in serum were measured and the rats were killed by cervical dislocation. Livers were harvested and weighed; hepatic left lobules were fixed with $10 \%$ formalin and pathological sections were prepared; hepatic right lobules were stored in liquid nitrogen. MDA, SOD, HL, and LPL content were measured in liver tissue.

$$
\text { Hepatic index }(\%)=[\text { hepatic wet weight }(\mathrm{g}) / \text { weight }(\mathrm{g})] \times 100 \quad \text { (Equation 1) }
$$

\section{Data processing}

Experimental data are reported as means \pm standard deviation. All data were analyzed by SPSS16.0; significance was analyzed by ANOVA. 


\section{RESULTS}

\section{Fatty liver model}

After being fed high-fat fodder for one week, rats in the model group had significantly increased levels of serum ALT, AST, TC, and TG, compared with rats of the control group (P< 0.01) (Table 1), suggesting the successful establishment of a rat fatty liver model (Jia et al., 2006).

Table 1. Level of blood lipid and liver function in rat after 1 week feeding on high-fat diet.

\begin{tabular}{l|l|l|l|l|l}
\hline Group & $\mathrm{N}$ & TC $(\mathrm{mM})$ & TG $(\mathrm{mM})$ & ALT(U/L) & AST (U/L) \\
\hline Control $^{\mathrm{a}}$ & 8 & $1.83 \pm 0.16$ & $0.47 \pm 0.08$ & $35.72 \pm 1.67$ & $87.11 \pm 2.23$ \\
\hline Model & 8 & $2.31 \pm 0.08^{\mathrm{b}}$ & $0.87 \pm 0.12^{\mathrm{b}}$ & $45.40 \pm 2.25^{\mathrm{b}}$ & $108.77 \pm 5.24^{\mathrm{b}}$ \\
\hline
\end{tabular}

aP $<0.05$ vs control group; ${ }^{\mathrm{b}} \mathrm{P}<0.01$ vs control group.

\section{Pathological changes of hepatic tissue in each group after PC treatment}

\section{Visual inspection}

The control group (Figure 1A) exhibited a red liver with a tough, lustrous and elastic surface and sharp edges; the model group (Figure 1B) showed liver volume significantly increased, and a cream-yellow liver with a tight capsule, blunt edges, focal yellow-white degeneration, and a greasy cut; the LG (Figure 1C) exhibited a dull-red liver with a soft, smooth surface and sharp edges. Compared with the model group, each $P$. eryngii cellulose dose group (Figure 1D-F) had different degrees of improvement, showing a dose-dependent relationship.
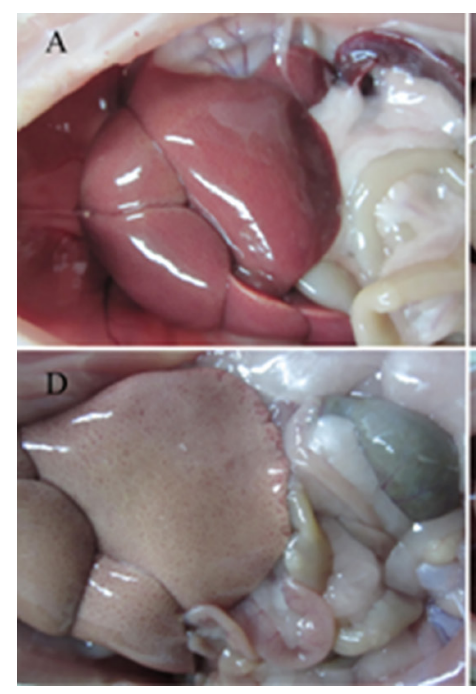
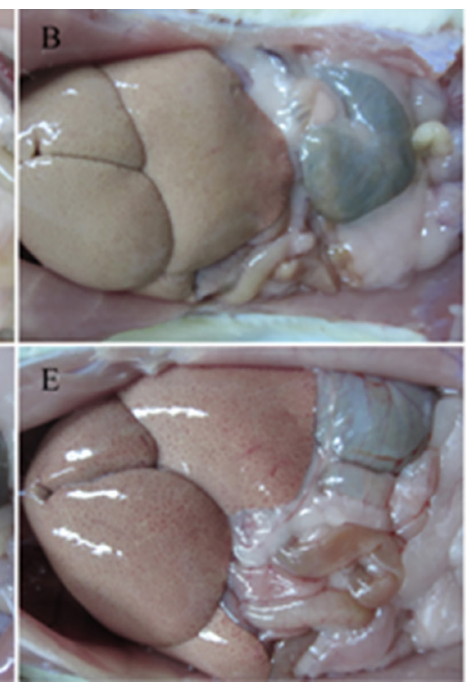

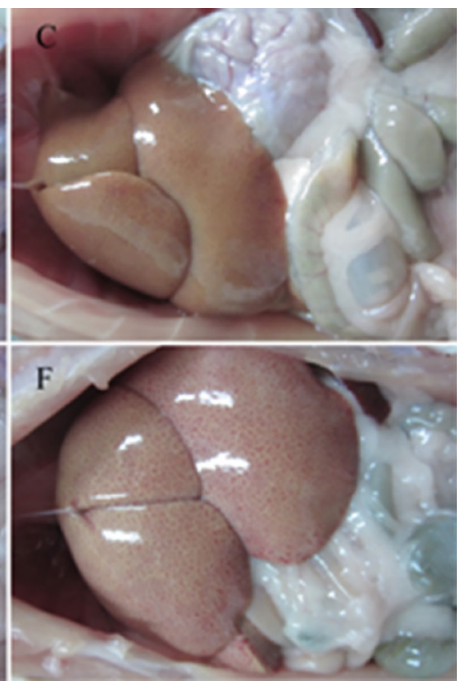

Figure 1. Appearance of liver in each group A. Control group; B. Model group; C. Lipitor group; D. Pleurotus eryngii cellulose low-dose group; E. Pleurotus eryngii cellulose middle-dose group; F. P. eryngii cellulose highdose group. 


\section{Histopathological observation}

In the control group (Figure 2A), the hepatic tissue structure was normal; hepatic cells appeared central vein-centered and arranged radially, with the hepatic sinusoid clearly visible and the hepatic cord arranged in neat rows. No fatty degeneration was apparent in the hepatic cells, nor were fat vacuoles present in the hepatic tissue. In the model group (Figure 2B), the hepatic lobule boundary was blurred, there was hepatic cord disorder, and the hepatic sinusoid disappeared. Extreme swelling resulted in round hepatic cells filled with fat vacuoles in the cytoplasm and severe fatty degeneration in the tissue. The LG (Figure 2C) had normal hepatic tissue structure and the hepatic cells appeared central vein-centered and arranged radially, without fatty degeneration and fat vacuoles, but swollen. The PCLG (Figure 2D) showed a blurred hepatic lobule boundary, hepatic cord disorder, hepatic cell swelling, and a few fat vacuoles in the cytoplasm. Compared with cells of the PCLG, the PCMG cells (Figure 2E) showed improved structure without fatty degeneration and fat vacuoles in hepatic cells. Compared with the results of the LG, the PCHG showed signs of recovery since only a few neutrophils appeared, but similar to that of the CG (Figure $2 \mathrm{~F}$ ).

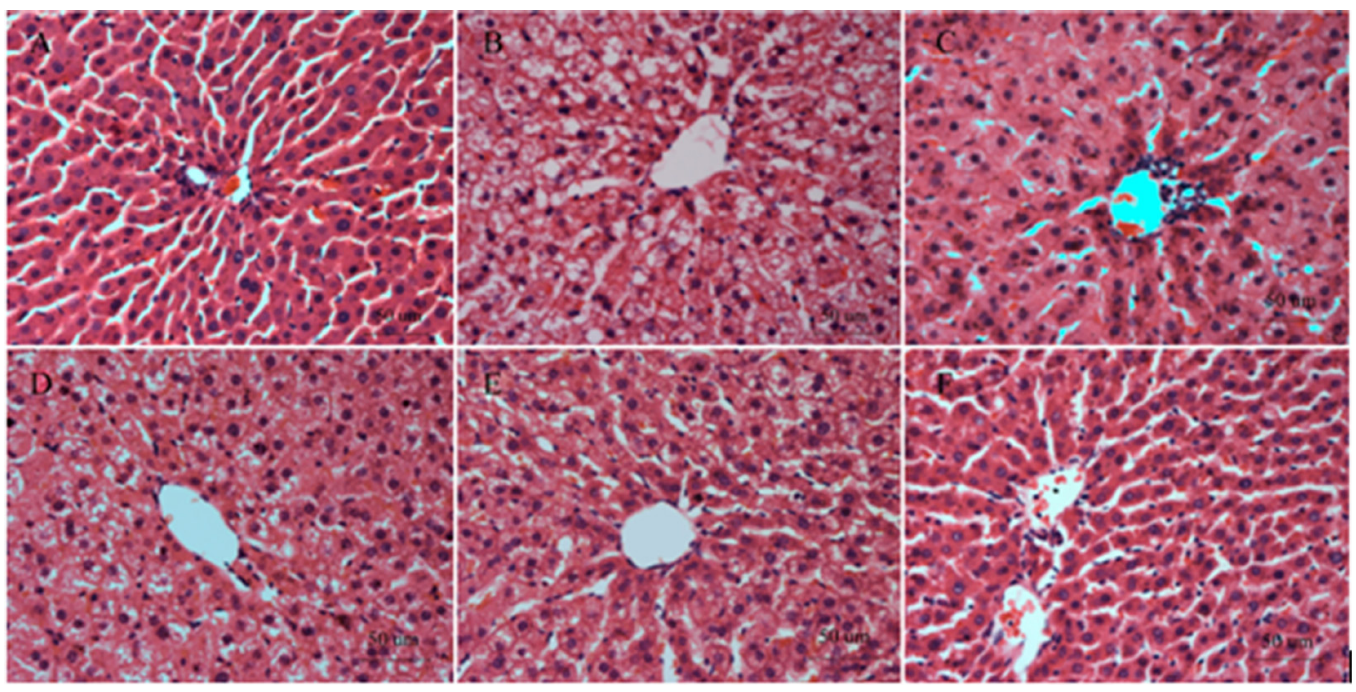

Figure 2. Histopathology of liver in each group (HE, 400X) A. Control group; B. model group; C. Lipitor group; D. Pleurotus eryngii cellulose low-dose group; E. P. eryngii cellulose middle-dose group; F. P. eryngii cellulose highdose group.

\section{Changes of blood lipids, hepatic function in rats after PC treatment}

After PC treatment for six weeks, compared with levels in the model group, blood lipid levels in rats of the PC treatment groups were very significantly decreased $(P<0.01)$. Table 2 shows a dose-related relationship: in the model group, TC level in serum was $2.56 \pm 0.16 \mathrm{mM}$; TC levels in the high-, middle-, and low-dose groups were $1.96 \pm 0.06,2.06 \pm 0.08$, and $2.19 \pm 0.09$ $\mathrm{mM}$, respectively. Hepatic function of the $\mathrm{PC}$ groups was also significantly decreased $(P<0.01)$; for the model group, serum ALT level was $92.23 \pm 10.6 \mathrm{U} / \mathrm{L}$, while ALT levels of the high-, middle-, and 
low-dose groups were 49.01 $\pm 4.17,59.33 \pm 6.23$, and $72.23 \pm 5.99 \mathrm{U} / \mathrm{L}$, respectively. Compared with levels in the LG, the TC and AST levels of the PCHG group showed no significant difference, with similar therapeutic effects.

\section{Table 2. Effect of PC on blood lipid and liver function of rats.}

\begin{tabular}{l|c|c|c|c|c}
\hline Group & $\mathrm{N}$ & TC $(\mathrm{mM})$ & TG $(\mathrm{mM})$ & ALT $(\mathrm{U} / \mathrm{L})$ & AST (U/L) \\
\hline CT $^{\mathrm{a}}$ & 8 & $1.81 \pm 0.12$ & $0.56 \pm 0.07$ & $39.30 \pm 2.36$ & $117.10 \pm 8.47$ \\
\hline MG & 8 & $2.56 \pm 0.16^{\mathrm{b}}$ & $1.51 \pm 0.06^{\mathrm{b}}$ & $92.23 \pm 10.6^{\mathrm{b}}$ & $208.36 \pm 19.33^{\mathrm{b}}$ \\
\hline LG & 8 & $1.89 \pm 0.09^{\mathrm{d}}$ & $0.86 \pm 0.09^{\text {bd }}$ & $44.08 \pm 2.61^{\text {bd }}$ & $139.03 \pm 6.06^{\text {bd }}$ \\
\hline PCHG & 8 & $1.96 \pm 0.06^{\text {bd }}$ & $1.00 \pm 0.09^{\text {bde }}$ & $49.01 \pm 4.17^{\text {bde }}$ & $143.46 \pm 9.16^{\text {bd }}$ \\
\hline PCMG & 8 & $2.06 \pm 0.08^{\text {bdf }}$ & $1.12 \pm 0.11^{\text {bd }}$ & $59.33 \pm 6.23^{\text {bdf }}$ & $157.78 \pm 10.96^{\text {bdf }}$ \\
\hline PCLG & 8 & $2.19 \pm 0.09^{\text {bdf }}$ & $1.28 \pm 0.05^{\text {bdf }}$ & $72.23 \pm 5.99^{\text {bdf }}$ & $180.00 \pm 7.54^{\text {bdf }}$ \\
\hline
\end{tabular}

aP < 0.05 vs control group (CT); ${ }^{\mathrm{b}} \mathrm{P}<0.01$ vs $\mathrm{CT}$; ${ }^{\mathrm{c} P}<0.05$; ${ }^{\mathrm{P}}<0.01$ vs model group (MG); $\mathrm{e} P<0.05$; $\mathrm{f}<0.01$ vs Lipitor group (LG).

\section{Changes of rat hepatic index and hepatic tissue-related enzyme activity after PC treatment}

Compared with activity seen in the model group, PC could significantly increase low SOD activity caused by lipid metabolism disorders, reduce high MDA content and high HL activity, reduce lipid deposition in hepatic tissue, and reduce hepatic indices (Table 3). Compared with activity seen in the model group, SOD activity in hepatic tissue of PC rats was significantly increased $(P<0.01)$, MDA was significantly decreased $(P<0.05)$, $H L$ activity was significantly decreased $(P<0.01)$, and hepatic index was significantly decreased $(P<0.05)$. However, the effect on MDA and LPL activity was not ideal (Table 3).

Table 3. Effect of PC on liver index, SOD, MDA, HL, LPL in rat liver.

\begin{tabular}{l|c|c|c|c|c|c}
\hline Group & $\mathrm{N}$ & SOD (U/mg prot) & MDA (nmol/mg prot) & HL (U/mg prot) & LPL (U/mg prot) & Liver index (\%) \\
\hline TG & 8 & $21.70 \pm 1.51$ & $14.35 \pm 1.48$ & $1.63 \pm 0.14$ & $1.46 \pm 0.25$ & $3.09 \pm 0.38$ \\
\hline MG & $8^{\mathrm{b}}$ & $9.29 \pm 1.79^{\mathrm{b}}$ & $40.14 \pm 3.07^{\mathrm{b}}$ & $2.62 \pm 0.18^{\mathrm{b}}$ & $0.85 \pm 0.05^{\mathrm{b}}$ & $4.56 \pm 0.61^{\mathrm{b}}$ \\
\hline LG & 8 & $18.23 \pm 2.60^{\text {bd }}$ & $16.69 \pm 1.26^{\mathrm{ad}}$ & $1.60 \pm 0.37^{\mathrm{d}}$ & $1.43 \pm 0.33^{\mathrm{d}}$ & $3.94 \pm 0.27^{\mathrm{ac}}$ \\
\hline PCHG & 6 & $15.87 \pm 1.50^{\text {bd }}$ & $35.36 \pm 4.52^{\text {bcf }}$ & $1.79 \pm 0.10^{\text {bd }}$ & $0.86 \pm 0.09^{\mathrm{a}}$ & $3.64 \pm 0.31^{\mathrm{ad}}$ \\
\hline PCMG & 7 & $14.10 \pm 2.83^{\mathrm{bde}}$ & $41.52 \pm 6.36^{\mathrm{bf}}$ & $1.98 \pm 0.21^{\text {bde }}$ & $0.71 \pm 0.05^{\mathrm{bcf}}$ & $4.02 \pm 0.29^{\mathrm{bc}}$ \\
\hline PCLG & 6 & $13.45 \pm 1.89^{\mathrm{bdf}}$ & $44.21 \pm 1.88^{\text {bcf }}$ & $2.06 \pm 0.25^{\text {bde }}$ & $0.46 \pm 0.06^{\mathrm{bf}}$ & $4.23 \pm 0.39^{\mathrm{b}}$ \\
\hline
\end{tabular}

${ }^{\mathrm{a} P}<0.05$, ${ }^{\mathrm{b}} \mathrm{P}<0.01$ vs control group; ${ }^{\mathrm{C}} \mathrm{P}<0.05$, ${ }^{\mathrm{d}} \mathrm{P}<0.01$ vs model group; ${ }^{\mathrm{e}} \mathrm{P}<0.05$, ${ }^{\mathrm{f}} \mathrm{P}<0.01$ vs Lipitor group.

\section{DISCUSSION}

Fatty liver disease is called fatty liver, and is related to heredity, environment, and metabolic stress . It is a pathological development of various liver diseases and is one of the most common diffuse liver diseases. The main pathological change is excessive accumulation of triglycerides in hepatocytes. Fatty liver is defined as an excessive accumulation of fat in liver cells, identified by either fat content exceeding $5 \%$ of liver weight or more than one-third liver cell fatty degeneration per unit area in histology (Lu et al., 2001; Li, 2013).

Liver is important in fat metabolism. When a body receives high-fat fodder, exogenous fat increases, TG formation from liver cell esterification increases, TG degradation in plasma decreases, 
and liver TG increases. Excessive intake of cholesterol and free fatty acids could cause cytotoxicity and liver cell dysfunction, reducing the capacity of liver cells to transport TG, resulting in abnormal liver function and abnormal increases in serum TC and TG (Dong and Wu, 2005; Yi et al., 2009). In this experiment, SD rats were given high-fat fodder for one week; compared with control group levels, TC, TG, ALT, and AST levels in serum of model groups were significantly increased $(P<0.01)$. After PC treatment for six weeks, blood lipid (TC and TG) levels in rats of each PC group was significantly decreased, liver function (ALT and AST) was significantly recovered. Therapeutic effect of PCHG was similar to that seen in the LG. Therapeutic effect of PC showed a dose-related relationship.

The exact pathogenesis of fatty liver is not yet defined, but is generally thought to involve increased hepatocyte fat synthesis and reduced oxidation. In this study, when compared with the control group, MDA content in hepatic tissue of the model group was significantly increased, SOD activity was significantly reduced $(P<0.01)$. We showed the rat hepatocyte oxygen consumption of the model group was significantly increased, numerous free radicals were produced in the body, and lipid peroxidation was enhanced, resulting in an increase of reaction product MDA. After PC treatment, SOD activity in hepatic tissue of each $P C$ group increased significantly $(P<0.01)$ and only PCHG inhibited an excessive increase of MDA $(P<0.05)$. We showed that $P C$ could eliminate free radicals and enhance antioxidant capacity and it could adjust and improve free radical metabolic balance.

LPL existed in blood capillary endothelial cells in extrahepatic tissue, could accelerate TG hydrolysis of catalytic chylomicron (CM) and very low-density lipoprotein (VLDL), reduced TG of lipid metabolism disorders in animals, and accelerated the degradation of CM and VLDL. In degradation of $C M$ and VLDL, surface lipid transferred to high-density lipoprotein (HDL), resulting in HDL2like particle formation. It benefited HDL increased, increased reverse cholesterol transportation, accelerated the transport of serum and extrahepatic cholesterol metabolism (Chen et al., 2004). HL, the key enzyme participating in endogenous triglyceride metabolism and lipoprotein metabolism, is synthesized by hepatocyte parenchymal cells, which only exist on the surface of hepatic endothelial cells. Increasing HL activity could cause lipoprotein metabolism changes, particularly a reduction in plasma HDL concentration, thereby slowing the reverse cholesterol transportation, leading to lipid metabolism disorders (Zhu et al., 2005). In this study, after performing PC treatment for six weeks, $\mathrm{HL}$ activity in rats of each $\mathrm{PC}$ group was significantly decreased $(P<0.01)$, while $L P L$ activity had no effect, significantly reduced hepatic index $(P<0.05)$, showed $P C$ can increase fat in hepatic metabolism, and reduced hepatic fat deposition.

In summary, PC enhanced antioxidant capacity of hepatic tissue, improved hepatic HL activity, and reduced hepatic fat deposition, thereby playing a role in hepatic protection and lowering lipid levels.

\section{ACKNOWLEDGMENTS}

Research supported by the Fujian Provincial Science and Technology Agency (\#2015N0030), the Fujian Province Natural Foundation of China (\#2013J01149), the Fujian Provincial Department of Education category B projects (\#JB11109), the Minnan Normal University Outstanding Young Research Personnel Training Projects (\#MJ13003).

\section{REFERENCES}

Chen W, Chen DW and Huang YQ (2004). Physiological function and specific expression of lipoprotein lipase. Chin. Anim. Husban. Vet. Med. 31: 29-30. 
Choi JH, Kim HG, Jin SW, Han EH, et al. (2013). Topical application of Pleurotus eryngii extracts inhibits 2,4-dinitrochlorobenzene-induced atopic dermatitis in NC/Nga mice by the regulation of Th1/Th2 balance. Food Chem. Toxicol. 53: 38-45.http://dx.doi.org/10.1016/j. fct.2012.11.025 PubMed

Dong CF and Wu GH (2005). Analysis of change in the lipid and liver function index in fatty liver patients. Lab. Med. 20: 160-161.

Ike K, Kameyama N, Ito A and Imai S (2012). Induction of a T-Helper 1 (Th1) immune response in mice by an extract from the Pleurotus eryngii (Eringi) mushroom. J. Med. Food 15: 1124-1128.http://dx.doi.org/10.1089/jmf.2012.2239 PubMed

Jia LC, Ma LX, Wu LP, Wang Y, et al. (2006). The interventional effect of balance diet on experimental fatty liver rat. Mod. Prev. Med. 33: 2254-2258.

Krupodorova T, Rybalko S and Barshteyn V (2014). Antiviral activity of Basidiomycete mycelia against influenza type A (serotype H1N1) and herpes simplex virus type 2 in cell culture. Virol. Sin. 29: 284-290.http://dx.doi.org/10.1007/s12250-014-3486-y PubMed

Lee C-Y, Park J-E, Kim B-B, Kim S-M, et al. (2009). Determination of mineral components in the cultivation substrates of edible mushrooms and their uptake into fruiting bodies. Mycobiology 37: 109-113.http://dx.doi.org/10.4489/MYC0.2009.37.2.109 PubMed

Li JW and Yang YX (2007). Review in Defining Dietary Fiber and Analysis Methods. Food Sci. 28: 350-354.

Li LP (2013). Application of statins in treatment with fatty liver disease. Drug Eval. 10: 21-24.

Li S and Shah NP (2014). Antioxidant and antibacterial activities of sulphated polysaccharides from Pleurotus eryngii and Streptococcus thermophilus ASCC 1275. Food Chem. 165: 262-270.http://dx.doi.org/10.1016/j.foodchem.2014.05.110 PubMed

Lu RJ, Ren DX and Yan M (2001). Progress in studies of the pathogenesis and therapy of the fatty liver. J. Gansu Sci. 13: 57-64.

Mizutani T, Inatomi S, Inazu A and Kawahara E (2010). Hypolipidemic effect of Pleurotus eryngii extract in fat-loaded mice. J. Nutr. Sci. Vitaminol. (Tokyo) 56: 48-53.http://dx.doi.org/10.3177/jnsv.56.48 PubMed

Mori K, Kobayashi C, Tomita T, Inatomi S, et al. (2008). Antiatherosclerotic effect of the edible mushrooms Pleurotus eryngii (Eringi), Grifola frondosa (Maitake), and Hypsizygus marmoreus (Bunashimeji) in apolipoprotein E-deficient mice. Nutr. Res. 28: $335-342$. http://dx.doi.org/10.1016/j.nutres.2008.03.010 PubMed

Shang X, Tan Q, Liu R, Yu K, et al. (2013). In vitro anti-Helicobacter pylori effects of medicinal mushroom extracts, with special emphasis on the Lion's Mane mushroom, Hericium erinaceus (higher Basidiomycetes). Int. J. Med. Mushrooms 15: 165-174.http://dx.doi. org/10.1615/IntJMedMushr.v15.i2.50 PubMed

Wang AN, Zhu HL, Wu LG, Wang AJ, et al. (2009). The function, modification and application of dietary fiber. J. Henan Univ. Technol. 30: 89-93.

Yi GY, Yang TS, Li YZ and Zhang WH (2009). The hepatic effects of combination therapy of probucol and atorvastatin in spontaneously hypertensive rats with high lipids diet. Chin. J. Arterioscler. 17: 117-121.

Zhu MH, Ding YS, Wu Y, An JL, et al. (2005). Prevention function of nicotinylsalicylic acid on experimental fatty liver in quails and relevant mechanism inquiry. J. Shihezi Univ. 23: 163-165. 